\title{
Prostaglandin E1 analog increases spinal cord blood flow at the point of compression during and after experimental spinal cord injury
}

\author{
Y Hamamoto, T Ogata, T Morino, M Hino and H Yamamoto \\ Department of Orthopaedic Surgery, Ehime University School of Medicine, Tohon City, Ehime, Japan
}

\begin{abstract}
Study design: An in vivo study using a spinal cord compression model in rats.
Objectives: To evaluate the effect of prostaglandin E1 (PGE1) on the change in thoracic spinal cord blood flow and on hind-limb motor function.

Background: Until now, effect of PGE1 on spinal cord blood flow the point of compression has not been tested.

Methods: Our newly developed blood flow measurement system was a combination of a noncontacttype Laser Doppler system and a spinal cord compression device. The rat thoracic spinal cord was exposed and spinal cord blood flow at the point of compression was measured before, during and after compression. The functioning of the animals' hind-limbs was evaluated by the BBB Scale and by measuring the frequency of voluntary standing.

Results: During the compression period, spinal cord blood flow was significantly higher in the PGE1treated rats than in the control rats, which did not receive PGE1. After decompression, the spinal cord blood flow rapidly recovered to about $60 \%$ of the precompression level in the control rats. When the animals were treated with PGE1, blood flow after decompression reached about $90 \%$ of the precompression level.

Twenty-gram compression for 40 mins induced motor deficiencies in the rat hind-limbs. The application of PGE1 significantly improved motor function of the rat hind-limbs after spinal cord injury.

Conclusions: The application of PGE1 increased spinal cord blood flow during and after spinal cord compression, and improved motor function after the spinal cord injury.

Spinal Cord (2010) 48, 149-153; doi:10.1038/sc.2009.99; published online 18 August 2009
\end{abstract}

Keywords: prostaglandin; blood flow; compression; motor deficiency

\section{Introduction}

Ischemia is one of the key events in the processes of neuronal degeneration after spinal cord injury. After mechanical damage such as an axonal tear or bleeding, degeneration processes including the release of excitatory amino acids, elevation of intracellular calcium ions, and induction of apoptosis follow tissue ischemia.

In the clinical field, most spinal cord ischemia is the result of spinal cord compression, due to conditions such as cervical spondylotic myelopathy, disc herniation, ossification of the posterior longitudinal ligament, tumors, infection and traumatic spinal cord injury.

Several previous studies suggest that spinal cord blood flow is one of the most important factors in motor and sensory function. Osenbach et al. ${ }^{1}$ reported that experimental rabbit

Correspondence: Dr T Ogata, Department of Orthopaedic Surgery, Ehime University, School of Medicine, Shitsukawa, Tohon-City, Ehime, 791-0295, Japan.

E-mail: ogata@m.ehime-u.ac.jp

Received 2 March 2009; revised 16 June 2009; accepted 25 June 2009; published online 18 August 2009 spinal cord ischemia caused by balloon occlusion of the infrarenal aorta decreased the amplitude of somatosensory and motor evoked potentials after reperfusion. Weir et al. ${ }^{2}$ reported that ischemia caused by aortic occlusion induced motor neuron loss in the lumbosacral spinal cord and paraplegia after reperfusion. As an acute treatment for spinal cord injury, maintenance of systolic blood pressure above $90 \mathrm{~mm} \mathrm{Hg}$ and mean arterial blood pressure between 85 and $90 \mathrm{~mm} \mathrm{Hg}$ is recommended for the first week to maintain spinal cord blood flow. ${ }^{3}$ Therefore, the restoration of blood circulation is a strong candidate to become a therapy for prevention of neuronal degradation after spinal cord injury.

Prostaglandin E1 (PGE1) is known to be a powerful vasodilator, ${ }^{4}$ and has been established as an effective therapeutic agent for the treatment of peripheral vascular diseases by enhancing blood supply. Recently, its effect on peripheral nerve diseases has been highlighted. The application of PGE1 is an effective therapy against intermittent claudication in patients with lumbar spinal canal stenosis. ${ }^{5}$ Komaba et al. ${ }^{6}$ reported that the intravenous application of 
PGE1 also increases cerebral blood flow in patients with chronic cerebral infarction. Konno et al. ${ }^{7}$ reported that a PGE1 analog increased the nerve-conduction velocity of the cauda equina in a canine experimental acute compression model.

To evaluate the effect of PGE1 on spinal cord compression injury, direct measurement of spinal cord blood flow at the point of compression is necessary. However, a method of real-time blood flow measurement at the point of compression has not been established to date. Here, we demonstrate a novel device for the measurement of rat spinal cord blood flow at the point of compression, and investigate the effect of PGE1 on spinal cord blood flow during and after mechanical compression.

In this study, we used lipo-PGE1, which incorporates a PGE1 analog (Alprostadil) into a lipid microsphere. It has been reported to efficiently accumulate in nervous tissue after intravenous injection and release prostaglandin. ${ }^{8}$

\section{Materials and methods}

\section{Measurement of rat spinal cord blood flow}

Our blood flow measurement system was a combination of a noncontact-type Laser Doppler system and a spinal cord compression device. ${ }^{9}$ The Laser Doppler probe was set in the weight of the spinal cord compression device. The tip of the probe, the part that contacts the dura, was covered with transparent soft silicone $3 \mathrm{~mm}$ in diameter and $5 \mathrm{~mm}$ in length (KE-1606, Shinetsu Chemical Co., Tokyo, Japan). The hardness of the silicone was 30 (durometer Scale A). The laser beam passed through the silicone, and blood flow was directly measured during spinal cord compression (Figure 1). The Laser Doppler blood flow meter (TBF-LN1, Unique Medical Co., Ltd, Tokyo, Japan) can measure blood flow within $3 \mathrm{~mm}$ of the point of contact. As our Laser Doppler system may also detect blood flow in the posterior part of the

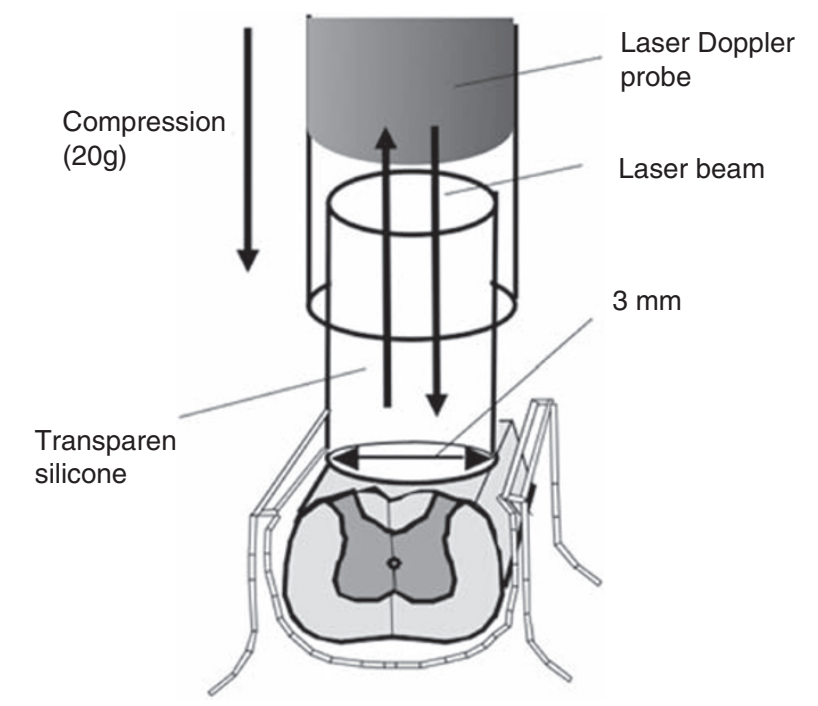

Figure 1 Schematic drawing of the real-time blood flow measurement system during compression. vertebral bone adjacent to the spinal cord, the blood flow measurement value in complete ischemic conditions was obtained by tying the spinal cord distal and proximal to the measurement site. We used this complete ischemic value as a baseline and subtracted this value from all of the measurement values. During the experiment, rat systemic blood pressure was monitored in the tail. No remarkable change in systemic blood flow was detected by spinal cord compression (data not shown).

\section{Animal model of spinal cord injury}

Female Wistar rats (250-300 g, obtained from Clea Japan, Tokyo, Japan) were used in this study. Under general anesthesia using halothane, the rat spinal cord was carefully exposed by removing the vertebral lamina at the 11th vertebra. The compression device including the Laser Doppler probe was gently attached to the surface of the dura. Immediately after attaching the device, no compression was applied and normal spinal cord blood flow was measured (precompression level). Then, blood flow was measured in every 5 mins. A 20-g weight was loaded for 40 mins to compress the spinal cord and create ischemic conditions, and then the weight was removed (decompression). We previously reported that a $20-\mathrm{g}$ weight produced complete ischemia in the rat experimental model. ${ }^{9}$ After decompression, blood flow was measured for another 30 mins. The blood flow data were expressed as percentages of the precompression level. In some animals, a laminectomy was performed without spinal cord compression (sham group, $n=13$ ). The research protocol was accepted by the ethical committee for animal experiments of Ehime University (Ehime, Japan).

\section{Infusion of Lipo-PGE1}

Lipo-PGE1 (10 $\mu \mathrm{g} \mathrm{kg}^{-1}$; Mitsubishi Pharma, Osaka, Japan) was injected intravenously 5 mins before compression (PGE1 group). In the saline group, the same amount of saline was injected in place of Lipo-PGE1.

\section{Evaluation of neurological recovery}

Hind-limb function was evaluated by using the BBB Scale ${ }^{10}$ and by measuring the standing frequency ${ }^{11}$ at 3,7 and 14 days after spinal cord compression. To measure standing frequency, we used Scanet MV-10 (MATYS Co., Ltd, Tokyo, Japan). Hind-limb function was assessed by measuring the frequency of vertical movements using the upper detector (13 cm above the floor). Rats often assume a posture in which their fore-limbs are raised and their weight is supported only on the hind-limbs. We call this vertical movement 'standing'. In this study, standing frequency was measured over a period of 30 mins.

Data analysis

For statistical analysis, Mann-Whitney's $U$-test was used. 


\section{Results}

The change in spinal cord blood flow during and after compression is shown in Figure 2. In the saline group, compression with a $20-\mathrm{g}$ weight reduced spinal cord blood flow from 100-2.8 $\pm 0.99 \%$ (average \pm s.e.m.) in the first 5 mins; blood flow reached its lowest level $(0.02 \pm 0.95 \%)$ within 10 mins after the $20 \mathrm{~g}$ compression. After decompression, spinal cord blood flow rapidly recovered to $60.6 \pm 6.97 \%$ within 5 mins. Then, the blood flow steadied at around $60 \%$ of the precompression level until 30 mins after decompression. In the PGE1 group, blood flow was also reduced from $100 \pm 12.1 \pm 2.78 \%$ by $20 \mathrm{~g}$ compression in the first 5 mins. However, the blood flow was significantly $(P<0.05)$ higher than that of the saline group through the compression period. After decompression, spinal cord blood flow recovered to $92.5 \pm 11.45 \%$ of the precompression level within the first 5 mins. The recovery of blood flow was significantly $(P<0.05)$ higher than that of the saline group. After the first 5 mins, the blood flow in the PGE1 group reached its highest level $(95.3 \pm 14.09 \%)$ within 10 mins after decompression, then gradually decreased. The blood flow in the PGE1 group finally steadied at around $88.0 \pm 12.1 \%$ of the precompression level 30 mins after decompression.

The motor function of the rat hind-limbs was evaluated by BBB Scale and by measuring standing frequency. Apparent motor deficiency was observed in the saline group 3 days after decompression $(10.5 \pm 2.27)$. At the same timepoint, the BBB Score in the PGE1 group $(17.9 \pm 1.24)$ was significantly higher $(P<0.01)$ than that of the saline group. As our spinal cord compression was not severe, motor function in both saline group $(19.1 \pm 0.67)$ and PGE1 group (20.2 \pm 0.55$)$ recovered by 7 days after decompression and no statistically significant difference between the BBB Scores of the saline and PGE1 groups was observed at 7 or 14 days after decompression (Figure 3).

Standing frequency is much more sensitive than the BBB Scale for assessing motor function after a mild injury. In the sham rats, which underwent laminectomy without decompression, the average standing frequency was $114 \pm 9.11$ times per 30 mins. Three days after decompression, the standing frequency in the saline group decreased to $9.80 \pm 3.82$ times per 30 mins and then gradually increased. The standing frequencies in the saline group in 7 and 14 days after decompression were $24.0 \pm 6.25$ and $56.8 \pm 5.81$ times per 30 mins, respectively. In the PGE1 group, the standing frequency was $28.3 \pm 7.78$ times per 30 mins 3 days after decompression, and then gradually increased until 14 days after decompression. The standing frequencies in the PGE1 group in 7 and 14 days after decompression were $47.7 \pm 8.01$ and $62.7 \pm 8.58$ times per 30 mins, respectively. At both 3 and 7 days after compression, the standing frequency in the PGE1 group was significantly higher $(P<0.05)$ than that of the saline group (Figure 4).

\section{Discussion}

To clarify the pathophysiology of spinal cord compression damage, monitoring of local blood flow in the compressed

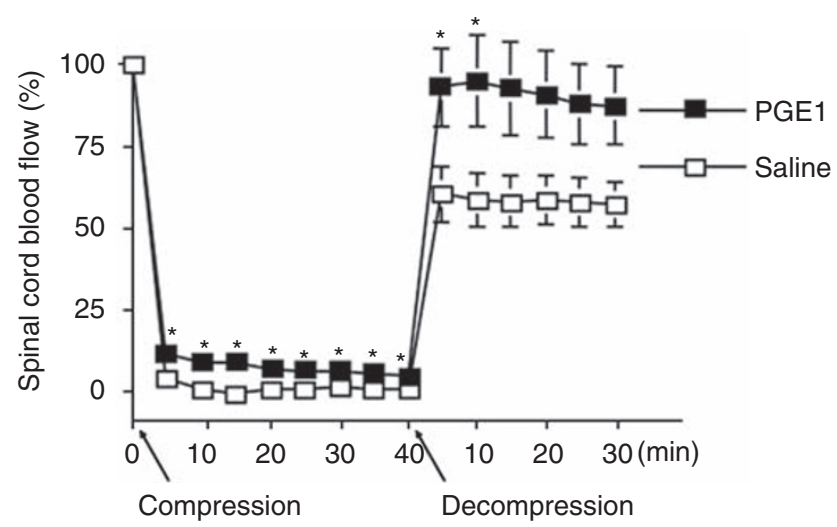

Figure 2 Spinal cord blood flow during compression and after decompression. Spinal cord blood flow was measured every 5 mins. After decompression, blood flow rapidly recovered in both groups. Data are expressed as the mean \pm s.e.m. $(n=10)$. Statistical differences between the two groups are represented by asterisks $\left({ }^{*} P<0.05\right)$.

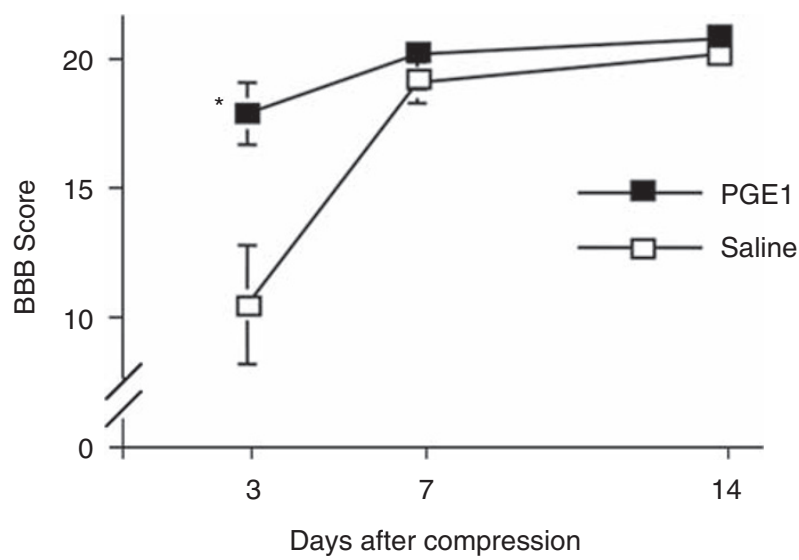

Figure 3 BBB Score after the compression injury. A 20-g weight was loaded for 40 mins. At 3, 7 and 14 days after compression, hindlimb motor function was evaluated by the BBB Scale. Data are expressed as the mean \pm s.e.m. $(n=10)$. Statistical differences between the PGE1 group and the saline group are represented by asterisks $\left({ }^{*} P<0.01\right)$.

spinal cord tissue is necessary. We have developed a system for measuring spinal cord blood flow at the point of compression. ${ }^{9}$ Using this system, we tried to measure spinal cord blood flow during and after spinal cord compression with or without lipo-PGE1 infusion. Kawamura et al. ${ }^{12}$ reported that apoptotic cell death in the dorsal horn induced by sciatic nerve occlusion was almost completely blocked by $10 \mu \mathrm{g} / \mathrm{kg}$ lipo-PGE1 injection. The lower dose ( 1 and $3 \mu \mathrm{g} / \mathrm{kg})$ of lipo-PGE1 showed partial inhibition of the apoptosis. Therefore, we used $10 \mu \mathrm{g} / \mathrm{kg}$ lipo-PGE1 in this study. It is possible that lower dose of lipo-PGE1 also showed some extent of increase of spinal cord blood flow and improvement of motor functions.

During the compression period, the group that received intravenous lipo-PGE1 showed significantly greater spinal cord blood flow at the point of compression compared with the saline group. The effect of lipo-PGE1 on blood flow was 


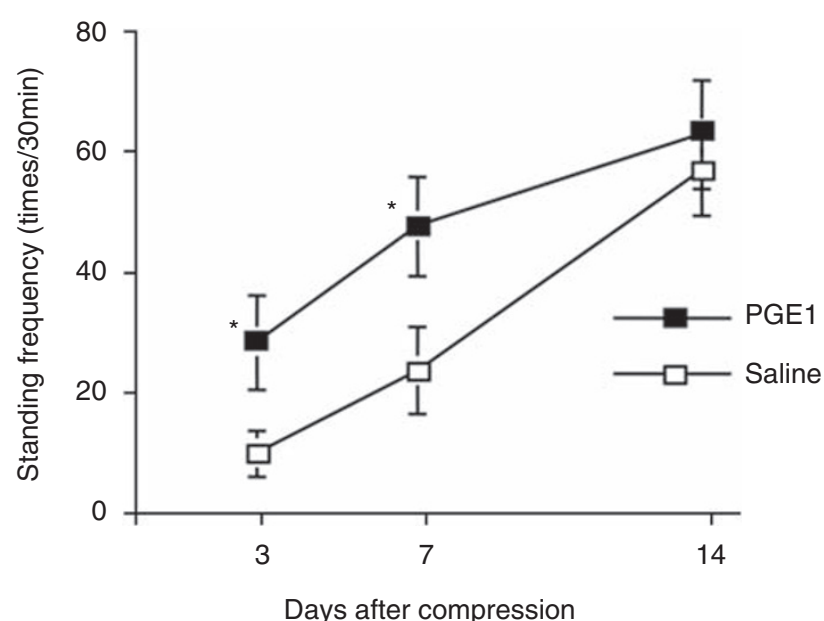

Figure 4 Standing frequency after the compression injury. A 20 -g weight was loaded for 40 mins. At 3,7 and 14 days after compression, hind-limb motor function was evaluated by measuring voluntary standing frequency. Data are expressed as the mean \pm s.e.m. $(n=10)$. Statistical differences between the PGE1 group and the saline group are represented by asterisks $\left({ }^{*} P<0.05\right)$.

even more remarkable after decompression. In the saline group, blood flow was about $60 \%$ of the precompression level. On the other hand, re-perfused blood flow in the PGE1 group was about $90 \%$ of the precompression level (Figure 2). Hind-limb motor recovery in the PGE1 group was better than that of the saline group (Figures 3 and 4).

The higher rate of blood flow caused by lipo-PGE1 may beneficially influence motor recovery. There are two possible working mechanisms for motor function recovery as a result of increased blood flow: (1) avoidance of complete ischemia during the compression period through the administration of PGE1 treatment and (2) higher blood supply after decompression as a result of PGE1 treatment. Both mechanisms may work together to help protect motor neurons from secondary neuronal damage.

Jacobs et al. ${ }^{13}$ reported that spinal cord ischemia induces progressive breakdown of blood-spinal cord barrier integrity. Thus in our study, it is possible that the application of PGE1 protected the blood-spinal cord barrier and allowed 90\% of the original blood flow to be maintained after the decompression. Several working mechanisms of PGE1 have been reported: (a) dilatation of the capillary wall, (b) inhibition of platelet aggregation, ${ }^{14}$ and (c) improved deformability of red blood cells. In addition, an inhibitory effect on superoxide production has been reported. ${ }^{15}$

After ischemia in the CNS, several type of immune cells such as neutrophils and microglia proliferate and are activated in the injured CNS tissue. ${ }^{16}$ These immune cells accelerate neuronal damage by releasing superoxide, nitric oxide and cytokines. These substances appear to be the targets of PGE1. Naruo et al. ${ }^{17}$ reported that PGE1 treatment reduces trauma-induced rat spinal cord injury by inhibiting neutrophil activation. Haynes et al. ${ }^{18}$ reported that a PGE1 analog, misoprostol, regulates inflammatory cytokines and immune functions in cultured T-cells or macrophages. Using cultured spinal cord microglia, we demonstrated earlier that the application of PGE1 inhibits the release of nitric oxide and TNF- $\alpha$ from activated microglia. ${ }^{19}$ It is possible that PGE1 protects spinal cord tissue including the blood-spinal cord barrier through inhibition of the toxic responses of these immune cells after spinal cord compression injury. After a compression injury, microglial proliferation and activation in the spinal cord tissue occur up to $72 \mathrm{~h}$ after compression. ${ }^{11}$ Grabits et al. ${ }^{20}$ reported that PGE1 protected the spinal cord when they applied it $1 \mathrm{~h}$ after aortic occlusion. These reports indicate that not only acute, but also delayed neuronal degradation caused by pathological inflammatory responses to spinal cord ischemia can be prevented by the application of PGE1.

In this study, using a novel device for blood flow measurement, we were able to observe real-time blood flow at the point of compression and observed an increase in the blood supply as a result of the application of intravenous PGE1 during and after spinal cord compression. Decrease of blood flow after spinal cord injury reduced the effect of systemic applied pharmacological agents such as methylprednisolone. If the PGE1 applies together with other drugs, there is a great advantage of drug delivery by increase of blood flow to the injury site. PGE1 is a candidate to be a therapeutic agent for several spinal cord compression diseases and injuries.

\section{References}

1 Osenbach RK, Hitchon PW, Mouw L, Yamada T. Effects of spinal cord ischemia on evoked potential recovery and postischemic regional spinal cord blood flow. J Spinal Disord 1993; 6: 146-154.

2 Weir CJ, Zivin JA, Lyden PD. Inter-relationships between spinal cord blood flow, neuronal death and nerurological function in rabbit spinal cord ischemia. Brain Res 2002; 946: 43-51.

3 Hadley MN, Walters BC, Grabb PA, Oyesiku NM, Przybylski GJ, Resnick DK et al. Guidelines for the management of acute cervical spine and spinal cord injuries. Clin Neurosurg 2002; 49: 407-498.

4 Carlson LA, Olsson AG. Intravenous prostaglandin E1 in severe peripheral vascular disease. Lancet 1976; 2: 810.

5 Scheffler P, Gross J, Markwirth T, Maier J, Schieffer H. Progress in the prostaglandin E1-therapy of the intermittent claudication by means of bolus injections of LIPO-prostaglandin E1 (LIPO-PGE1). Eur J Clin Pharmacol 1996; 51: 235-239.

6 Komaba Y, Kitamura S, Terashi A. Effect of prostaglandin E1 on cerebral blood flow in patients with chronic cerebral infarction. Intern Med 1998; 37: 841-846.

7 Konno S, Kayama S, Olmarker K, Kikuchi S. Effects of OP-1206 (prostaglandin E1) on nerve-conduction velocity in the dog cauda equina subjected to acute experimental compression. J Spinal Disord 1996; 9: 103-106.

8 Mizushima Y, Yanagawa A, Hoshi K. Prostaglandin E1 is more effective, when incorporated in lipid microspheres for treatment of peripheral vascular diseases in man. J Pharm Pharmacol 1983; 35: 667-668.

9 Hamamoto Y, Ogata T, Morino T, Hino M, Yamamoto H. Realtime direct measurement of spinal cord blood flow at the site of compression: relationship between blood flow recovery and motor deficiency in spinal cord injury. Spine 2007; 32: 1955-1962.

10 Basso DM, Beattie MS, Bresnahan JC. Graded histological and locomotor outcomes after spinal cord contusion using the NYU weight-drop device versus transection. Exp Neurol 1996; 139: 244-256.

11 Morino T, Ogata T, Horiuchi H, Takeba J, Okumura H, Miyazaki T et al. Delayed neuronal damage related to microglia proliferation after mild spinal cord compression injury. Neurosci Res 2003; 46: 309-318. 
12 Kawamura T, Akira T, Watanabe M, Kagitani Y. Prostaglandin E1 prevents apoptotic cell death in superficial dorsal horn of rat spinal cord. Neuropharmacology 1997; 36: 1023-1030.

13 Jacobs TP, Kempski O, McKinley D, Dutka AJ, Hallenbeck JM, Feuerstein G. Blood flow and vascular permeability during motor dysfunction in a rabbit model of spinal cord ischemia. Stroke 1992; 23: 367-373.

14 Kaiya H. Prostaglandin E1 suppression of platelet aggregation response in schizophrenia. Schizophr Res 1991; 5: 67-80.

15 Miyata M, Ajima H, Kondo Y, Kanno T, Ito M, Nakahata N et al. Concomitant use of prostaglandin E1 and prednisolone; inhibition of superoxide anion production by polymorphonuclear leucocytes. Fukushima J Med Sci 1995; 41: 15-28.

16 Morioka T, Kalehua AN, Streit WJ. The microglia reaction in the rat dorsal hippocampus following transient forebrain ischemia. J Cereb Blood Flow Metab 1991; 11: 966-973.
17 Naruo S, Okajima K, Taoka Y, Uchiba M, Nakamura T, Okabe H et al. Prostaglandin E1 reduces compression trauma-induced spinal cord injury in rats mainly by inhibiting neutrophil activation. J Neurotrauma 2003; 20: 221-228.

18 Haynes DR, Whitehouse MW, Vernon-Roberts B. The prostaglandin E1 analogue, misoprostol, regulates inflammatory cytokines and immune functions in vitro like the natural prostaglandins E1, E2 and E3. Immunology 1992; 76: 251-257.

19 Chuai M, Ogata T, Morino T, Okumura H, Yamamoto $H$, Schubert P. Prostaglandin E1 analog inhibits the microglia function: suppression of lipopolysaccharide-induced nitric oxide and TNF- $\alpha$ release. J Orthopedic Res 2002; 20: 1246-1252.

20 Grabits K, Freye E, Prior R, Braun M. Protection from spinal cord injury by intravenous prostaglandin E 1 PGE1) after one hour occlusion of the deacending thoracic aorta. Prostaglandins Clin Res 1989; 301: 211-216. 